\title{
Parasitos gastrintestinais em fezes de gatos domiciliados no município de Pelotas, RS, Brasil
}

Received: oct, 2019; Accepted: sep, 2020

\author{
Alexsander Ferraz ${ }^{1^{*}}$, Bruna dos Santos Pires ${ }^{1}$, Eugênia Tavares Barwaldt ${ }^{1}$, \\ Eduarda Machado dos Santos ${ }^{1}$, Camila Moura de Lima ${ }^{1}$, \\ Tanize Angonesi de Castro ${ }^{2}$, Márcia de Oliveira Nobre ${ }^{1}$, \\ Leandro Quintana Nizoli ${ }^{2}$
}

\begin{abstract}
Resumo: Este estudo, teve como objetivo, determinar a frequência de ovos, cistos e oocistos de parasitos gastrintestinais em gatos domésticos no município de Pelotas, RS, Brasil. Foram analisadas 60 amostras fecais, destas, $43(71,7 \%)$ eram procedentes de animais adultos e $17(28,3 \%)$ de filhotes. O material foi processado pelas técnicas de Willis \& Mollay (1921), Faust (1938) e Hoffmann, Pons e Janer (1934). Destas amostras, 22 $(36,7 \%)$ foram positivas para pelo menos um gênero de parasito, sendo 17 $(77,3 \%)$ com contaminaçao simples e $5(22,7 \%)$ com associação de parasitos. Ovos de parasitos do gênero Ancylostoma e Toxocara foram os mais observados, estando presentes em 8 amostras cada (13,3\%). Nos animais jovens, houve maior frequência de Toxocara spp., enquanto nos adultos, predominou Ancylostoma spp. Foram observados ainda, ovos dos cestódeos, Dipylidium caninum, Taenia Taeniaeformis e Spirometra sp., além de cistos de Giardia spp. e oocistos de Cystoisospora spp. A ocorrência de um número significativo de parasitos e a proximidade entre os animais e o homem mostra a necessidade de um controle mais efetivo para que ocorra a redução da carga parasitária dos animais e consequentemente do ambiente.
\end{abstract}

Palavras-chave: Felinos, diagnóstico, helmintos, protozoários

\section{Introdução}

Os animais domésticos, com destaque os felinos, estão convivendo progressivamente com os seres humanos por meio de vínculo afetivo e companhia, ajudando no bem-estar físico e psicológico (GÓMEZ et al., 2007; LIMA \& LUNA, 2012). A personalidade e comportamento dos gatos se

\footnotetext{
${ }^{1}$ Faculdade de Veterinária da Universidade Federal de Pelotas - Departamento de Clínicas Veterinária.

${ }^{2}$ Faculdade de Veterinária da Universidade Federal de Pelotas - Departamento de Veterinária Preventiva.

*Corresponding author: xanderferraz@yahoo.com.br, Faculdade de Veterinária - Universidade Federal de Pelotas - Campus Capão do Leão, s/n.
} 
ajustam à rotina da população moderna, consequentemente a um número gradativo de adeptos a essa espécie (MCPHERSON, 2005; COELHO et al., 2009; GENARO, 2010). Assim, segundo um levantamento realizado pelo IBGE (Instituto Brasileiro de Geografia e Estatística), a população de felinos em domicílios brasileiros foi estimada em 22,1 milhões, o que corresponde aproximadamente 1,9 gato doméstico por domicílio (IBGE, 2015).

Com a crescente presença dos felinos no cotidiano dos seres humanos são necessários cuidados com a saúde dos animais, uma vez que podem acarretar em uma fonte de agentes causadores de zoonoses, como os helmintos Ancylostoma spp., Toxocara spp., os protozoários Giardia spp. e Cryptosporidium spp. e o cestódeo Dipylidium caninum (HUBER et al., 2002; RAGOZO et al., 2002; ISHIZAKI et al., 2006; FUNADA et al., 2007; PALUDO et al., 2007; TORRICO et al., 2008).

Dentre as enfermidades mais frequentes que acometem os felinos ressaltam-se as parasitoses gastrintestinais (MCCARTHY \& MOORE, 2000; ISHIZAKI et al., 2006). Sendo a ocorrência não influenciada pela raça, idade ou sexo; no entanto, os gatos machos não castrados, em decorrência do comportamento da espécie, realizam deslocamentos a procura de fêmeas para acasalamento e para demarcação territorial (MIRCEN et al., 2010; SOUSA et al., 2010; SANTARÉM et al., 2012). Consequentemente, ficam mais predispostos à contaminação com ovos de helmintos e oocistos de protozoários presentes nas fezes no ambiente (WEESE et al., 2002; ISHIZAKI et al., 2006; COELHO et al., 2009; MIRCEN et al., 2010).

As endoparasitoses interferem negativamente no desenvolvimento dos felinos e acarretam em ação espoliativa aos hospedeiros ( MCCARTHY \& MOORE, 2000; TORRICO et al., 2008). Os sinais clínicos apresentados pelo animal parasitado podem variar de assintomático até quadros graves, como: diarreia, êmese, redução de peso e dores abdominais (LEE et al., 2010). Sendo mais observados em filhotes, em felinos que vivem aglomerados 
(gatil) e em animais imunocomprometidos (BOWMAN \& LYNN, 2003; FUNADA et al., 2007; THOMPSON et al., 2007).

Em relação aos principais endoparasitos com potencial zoonótico transmitidos pelos felinos destacam-se: a larva migrans cutânea (LMC) causada pela migração de larvas infectantes de Ancylostoma spp. (RAGOZO et al., 2002; MORO et al., 2008), desencadeando uma inflamação cutânea autolimitante no homem (CASTRO et al., 2002; COELHO et al., 2009); a larva migrans visceral (LMV) ocasionada pela migração das larvas de Toxocara spp. nos tecidos (RAGOZO et al., 2002), as quais geram lesões inflamatórias nas vísceras dos seres humanos (MCCARTHY \& MOORE, 2000; SANTARÉM et al., 2004).

Além do protozoário Giardia spp. que acarreta a Giardíase (HUBER et al., 2002; FUNADA et al., 2007; THOMPSON et al., 2007; PALMER et al., 2008), responsável por surtos em gatos mediante a ingestão de água contaminada com cistos infectantes (COELHO et al., 2009).

Assim, fica evidente que animais parasitados são uma importante fonte de risco à saúde humana, em decorrência da contaminação de crianças e adultos, através da penetração ativa de larvas infectantes, ingestão de ovos e cistos eliminados nas fezes (MCCARTHY \& MOORE, 2000; JAFFRY et al., 2009). Em suma, é fundamental o conhecimento da frequência de parasitos gastrintestinais em animais de companhia como os felinos, uma vez que possuem caráter zoonótico e também para garantir a saúde e o bem estar destes animais (WEESE et al., 2002).

O objetivo deste trabalho foi determinar a presença de parasitos gastrintestinais, em amostras de fezes de gatos domiciliados no município de Pelotas, Rio Grande do Sul, Brasil. 


\section{Material e métodos}

Neste estudo, foram processadas e analisadas, durante o ano de 2018, 60 amostras fecais de gatos domiciliados no município de Pelotas, RS, destes, $43(71,7 \%)$ eram adultos e 17 (28,3\%) filhotes. A coleta do material foi realizada pelo próprio tutor, acondicionada em pote plástico, mantida sob refrigeração e encaminhada para o Laboratório de Doenças Parasitárias (Ladopar), da Faculdade de Veterinária (FAVet) da Universidade Federal de Pelotas (UFPel).

As técnicas utilizadas para análise foram: Willis Molay (1921), de caráter qualitativo e que possui o princípio da flutuação de ovos leves e oocistos em solução hipersaturada; Método de Faust (1938), técnica de centrifugo-flutuação em solução de sulfato de zinco a 33\%, utilizado para pesquisa de cistos de Giardia spp. e oocistos; e o método de Hoffman, Pons e Janer (1934), que consiste na sedimentação espontânea, utilizada para pesquisa de ovos pesados de helmintos, como cestoides e trematódeos. A leitura das lâminas foi realizada em microscópio óptico, com aumento de 100x e 400x. A identificação dos gêneros de parasitos foi realizada a partir das características morfológicas dos ovos, oocistos e cistos.

\section{Resultados}

Das amostras analisadas, constatou-se que 22 (36,7\%) estavam positivas para algum gênero de parasito, destas, 17 (77,3\%) apresentavam infecção simples e $5(22,7 \%)$ associação de parasitos. Os gêneros mais prevalente foram Ancylostoma e Toxocara, presentes em 8 amostras cada (13,3\%). Foram observados ainda, ovos dos cestoides Dipylidium caninum, Spirometra sp. e Taenia taeniaeformis, cistos de Giardia spp. e oocistos de Cystoisospora spp. (Tabela 1). 
Analisando a frequência por idade, constatou-se que $29,4 \%$ dos adultos e $42,9 \%$ dos filhotes estavam parasitados. Nos felinos adultos, Ancylostoma spp. foi o parasito encontrado em maior número de amostras, sendo observado em 16,7\% (7/43), nos filhotes, o gênero Toxocara, presente em $35,2 \%$ das amostras (6/17), foi o mais prevalente. A distribuição dos gêneros por idade, está demonstrada na Tabela 2.

Tabela 01: Frequência de parasitos gastrintestinais em fezes de gatos domiciliados no município de Pelotas, Rio Grande de Sul, Brasil, analisadas durante o ano de 2018.

\begin{tabular}{cccc}
\hline Parasito & Frequência & \% positivos (22) & \% total (60) \\
\hline Ancylostoma spp. & 08 & 36,4 & 13,3 \\
Toxocara sp. & 08 & 36,4 & 13,3 \\
Cystoisospora spp. & 07 & 31,8 & 11,7 \\
Dipylidium caninum & 04 & 18,2 & 6,7 \\
Giardia spp. & 04 & 18,2 & 6,7 \\
Taenia & 02 & 9,1 & 3,3 \\
Taeniaeformis & & & 1,7 \\
Spirometra sp. & 01 & 4,5 & \\
\hline
\end{tabular}

Tabela 02: Frequência de parasitos gastrintestinais em fezes de gatos domiciliados no município de Pelotas, Rio Grande de Sul, Brasil, analisadas durante o ano de 2018, por idade.

\begin{tabular}{ccccc}
\hline Parasito & Adultos (n.43) & \multicolumn{3}{c}{ Filhotes (n.17) } \\
\hline & Amostras Positivas & $\%$ & Amostras Positivas & $\%$ \\
\hline Ancylostoma spp. & 07 & 16,3 & 01 & 5,9 \\
Toxocara spp. & 02 & 4,7 & 06 & 35,2 \\
Cystoisospora spp. & 04 & 9,3 & 03 & 17,6 \\
Dipylidium & 04 & 9,3 & - & - \\
caninum & & & & - \\
Giardia spp. & 04 & 9,3 & - & - \\
Taenia & 02 & 4,6 & - & - \\
Taeniaeformis & & & & - \\
Spirometra sp. & 01 & 2,3 & & \\
\hline
\end{tabular}




\section{Discussão}

No presente estudo, verificou-se que 36,7\% das amostras analisadas (22/60), estavam positivas para algum gênero de parasito. Marques et al. (2017), na cidade de Porto Alegre/RS, utilizando as mesmas técnicas diagnósticas, encontraram parasitismo em $31,85 \%$ das amostras, percentual próximo ao observado no presente estudo. Em contrapartida, Pivoto et al. (2013), em Santa Maria/RS, relataram que 47,1\% dos felinos avaliados apresentaram algum gênero de enteroparasito, e Silva et al. (2017), examinando o mesmo número de amostras fecais (60), obtiveram $65 \%$ de gatos positivos no Maranhão, valor expressivamente maior. Valores inferiores foram descritos por Lima et al. (2018) (17,0\%) e Palmer et al. (2008), com 17,0\% e 18,4\%, respectivamente.

Pelos hábitos e comportamentos peculiares, os gatos podem contribuir para a disseminação de determinados parasitos como, por exemplo, Ancylostoma spp. e Toxocara spp. (Mundim et al., 2004). O Ancylostoma spp., responsável pela Ancilostomiase em cães e gatos, foi encontrado em $8 / 60$ amostras (13,3\%), podendo causar quadros de diarreia e anemia, pois trata-se de um parasito hematófago. Nos humanos é o agente etiológico da zoonose parasitária denominada Larva Migrans Cutânea (LMC), dermatite causada pela penetração e migração de larvas de terceiro estágio (forma infectante), provocando lesões serpinginosas, com aspecto de mapa, por isso é comumente chamada de bicho geográfico, sendo as mãos, pés, pernas e nádegas as partes do corpo mais afetadas pelo contato com solos arenosos contaminados (NEVES, 2005; REY, 2008). Este foi o gênero mais frequente nos gatos adultos (16,3\%). Essa maior ocorrência pode ser explicada pelo fato dos gatos não desenvolverem imunidade contra antígenos de Ancylostoma spp., podendo infectar-se por toda a vida (BOAG et al., 2003).

Toxocara spp. também foi observado em 8/60 amostras (13,3\%), sendo mais frequente nos filhotes (35,2\%). Este parasito é responsável pela 
zoonose conhecida como Toxocariase, que ocorre pela ingestão acidental de ovos embrionados. Os sinais clínicos da enfermidade decorrem da reação inflamatória ocasionada pela migração do parasito, de acordo com o órgão afetado, sendo classificada em: Larva migrans visceral (LMV), Larva migrans ocular (LMO), oculta ou atípica e assintomática (HARALAMBIDOU et al., 2005; SAPORITO et al., 2008; ABOUGRAIN et al., 2009). Nos animais, os filhotes são mais acometidos, o que pode ser explicado pelo fato da transmissão lactogênica e transplacentária serem as principais fontes de infecção, e também pelo sistema imunológico imaturo durante essa faixa etária (BOWMAN et al., 2003). O período de maior ocorrência desta parasitose se dá logo após o nascimento, devido à eliminação das larvas através do leite materno de fêmeas infectadas, mesmo aquelas tratadas recentemente com anti-helmíntico e apresentem fezes com contagem de ovos negativa (BOWMAN et al. 2003). Estes fatores explicam a maior ocorrência deste gênero nos filhotes, observada no nosso estudo.

Cystoisospora spp. foi identificado em 7/60 amostras (11,7\%). Alguns estudos, como os de Pivoto et al. (2013); Marques et al. (2017); Silva et al. (2017); Monteiro et al. (2016); Dall'Agnol (2010) e Leite (2012) encontraram variação de $3,1 \%$ a $52,6 \%$ na frequência deste parasito. Este protozoário é responsável por quadros de diarreia e má absorção, sendo frequentemente observado na rotina da clínica de pequenos animais (BARUTZKI et al., 2013).

Cápsulas ovígeras de Dipylidium caninum estavam presentes em 4/60 amostras (6,7\%). Valores superiores (54,5\%), foram encontrados por Silva et al. (2001) em São Luís/MA, e inferiores, por Marques et al. (2017) e Monteiro et al. (2016), com 3,2\% e 0,88\%, respectivamente. A infecção por este cestoide ocorre através da ingestão acidental de pulgas e piolhos (hospedeiros intermediários), contendo a larva infectante deste parasito. Geralmente essa parasitose não representa grandes perigos para a saúde do animal, contudo, se a carga parasitária for elevada, pode acarretar irritação 
e inflamação da mucosa intestinal, e prurido na região perianal devido à saída ativa de proglotes através do ânus (LEITE, 2012).

O protozoário intestinal, Giardia spp., foi encontrado em 4/60 amostras (6,7\%). Pivoto et al. (2013), descreveram uma ocorrência de 4,2\% em gatos domiciliados em Santa Maria/RS, Silva et al. (2017), observaram este protozoário em 7,7\% dos gatos domiciliados atendidos em um Laboratório Veterinário em São Luís/MA e Vital et al. (2012) não detectou a presença do parasito durante as avaliações, porém o estudo considerou apenas 8 amostras de gatos domiciliados, o que pode ter contribuído para o resultado negativo. Este parasito possui potencial zoonótico e a infeç̧ão nos animais e no homem ocorre através da ingestão de cistos infectantes, presentes em água ou alimentos contaminados (MUNDIM et al., 2003)

A Taenia taeniaeformis, presente em apenas duas das amostras analisadas (3,3\%), tem como hospedeiros intermediários, pequenos roedores, que são infectados ao ingerir ovos presentes no ambiente, com patência que pode variar de vários meses a anos (ESCCAP, 2010). Resultado semelhante foi descrito por Mundim et al. (2004), que observaram uma frequência de 4\%, em Uberlândia, Minas Gerais.

O cestoide Spirometra sp. estava presente nas fezes de 1,7\% (2/60) dos gatos. Pivoto et al. (2013), Marques et al. (2017) e Dall' Agnol et al. (2010), relataram frequência de $0,5 \%, 0,92 \%$ e $2,6 \%$ deste parasito, respectivamente, enquanto Mundim et al. (2004) descreveram 4\% de positividade, porém utilizando animais necropsiados.

\section{Conclusão}

A partir do resultado obtido neste estudo, conclui-se que há ocorrência de um número elevado de gêneros de parasitos gastrintestinais nas amostras fecais dos gatos domiciliados do município de Pelotas, RS. Estes dados evidenciam a importância de um controle mais efetivo para que ocorra 
a redução da carga parasitária dos gatos e consequentemente do ambiente, reduzindo a exposição dos animais e do homem a estes parasitos, pois alguns gêneros apresentam potencial zoonótico.

\title{
Gastrintestinal parasites in feces of domestic cats in the municipality of Pelotas, RS, Brazil
}

\begin{abstract}
This study aimed to determine the frequency of eggs, cysts and oocysts of gastrointestinal parasites in domestic cats in the municipality of Pelotas, RS, Brazil. Sixty fecal samples were analyzed, of which $43(71.7 \%)$ were from adult animals and 17 (28.3\%) from puppies. The material was processed using the techniques of Willis \& Mollay (1921), Faust (1938) and Hoffmann, Pons and Janer (1934). Of these samples, 22 (36.7\%) were positive for at least one parasite genus, 17 (77.3\%) with simple contamination and 5 (22.7\%) with parasite association. Eggs from parasites of the genus Ancylostoma and Toxocara were the most observed, being present in 8 samples each (13.3\%). In young animals, there was a higher frequency of Toxocara spp., While in adults, Ancylostoma spp. Were also observed, eggs of the cestodes, Dipylidium caninum, Taenia Taeniaeformis and Spirometra sp., besides cysts of Giardia spp. and oocysts of Cystoisospora spp. The occurrence of a significant number of parasites and the proximity between animals and man shows the need for more effective control in order to reduce the parasitic load on the animals and consequently the environment.
\end{abstract}

Keywords: Felines, diagnosis, helminths, protozoa.

\section{Referências}

ABOUGRAIN, A.K.; NAHAISI, M.H.; MADI, N.S; SAIED, M.M.; GHENGHESH, K.S. Parasitological contamination in salad vegetables in Tripoli-Libya. Future Child, v.21, n.5, p.760-762, 2009. https://doi.org/10.1016/j.foodcont.2009.11.005

BARUTZKI, D.; SCHAPER, R. Dependant Prevalence of Endoparasites in Young Dogs and Cats up to One Year of Age. Parasitology Research., v.112, .1, p.119-131, 2013. https://doi.org/10.1007/s00436-013-3286-6 
BOAG, P. R.; PARSONS, J.C.; PRESIDENTE, P.J.; SPITHILL, T.J.; SEXTON, J.L. Characterisation of humoral imune responses in dogs vaccinated with irradiated Ancylostoma caninum. Veterinary Immunology and Immunopathology, v.92, n.1-2, p.87-94, 2003. https://doi.org/10.1016/S0165-2427(03)00006-0

BOWMAN, D.D.; LYNN, R.C. Georgis: Parasitologia para Veterinários. Elsevier Science, St. Louis, EUA, 2003.

CASTRO, C.S.V.; CERDEIRA, M.V.; MARTÍN, M.L.P. Larva migrans cutânea: diagnóstico de sospecha y tratamiento en Atención Primaria. MEDIFAM, v.12, n.10, p.655-657, 2002. https://doi.org/10.4321/S1131-57682002001000008

COELHO, W.M.D.; AMARANTE, A.F.T.; SOUTELLO, R.V.G.; MEIRELES, M.V.; BRESCIANI, K.D.S. Ocorrência de parasitos gastrintestinais em amostras fecais de felinos no município de Andradina. Revista Brasileira de Parasitologia Veterinária, v.18, n.2, p.46-49, 2009. https://doi.org/10.4322/rbpv.01802010

DALL' AGNOL, L.P.; OTTO, M.A.; SILVA, A.S.; MONTEIRO, S.G. Parasitos gastrintestinais em gatos naturalmente infectados no município de Santa Maria no estado do Rio Grande do Sul, Brasil. Acta Veterinaria Brasilica, v.4, n3, p.181-184, 2010 .

ESCCAP (Consejo Europeu para el Control de las Parasitosis de los Animales de Compañia), Guia ESCCAP no - Ectoparásitos Control de Insectos y Garrapatas que Parasitan a Perros y Gatos (2010).

FAUST, E.C.; D'ANTONI, J.S.; ODOM, V. A critical study of clinical laboratory technics for the diagnosis of protozoan cysts and helminth eggs in feces I. Preliminary communication. American Journal of Tropical Medicine, v18, n.2, p.169-183, 1938. https://doi.org/10.4269/ajtmh.1938.s1-18.169 
FERREIRA, F.P.; DIAS, R.C.F.; MARTINS, T.A.; CONSTANTINO, C.; PASQUALI, A.K.S.; VIDOTTO, O.; FREIRE, R.L.; NAVARRO, I.T. Frequência de parasitas gastrointestinais em cães e gatos do município de Londrina, PR, com enfoque em saúde pública. Semina: Ciências Agrárias,v.34, n.6, supl. 2, p.3851-3858, 2013. https://doi.org/10.5433/1679-0359.2013v34n6Supl2p3851

FUNADA, M.R.; PENA, H.F.J.; SOARES, R.M.; AMAKU, M.; GENNARI, S.M. Frequência de parasitos gastrintestinais em cães e gatos atendidos em hospitalescola veterinário da cidade de São Paulo. Arquivo Brasileiro de Medicina Veterinária e Zootecnia,v.59, n.5, p.1338-1340, 2007. https://doi.org/10.1590/S0102$\underline{09352007000500038}$

GENARO, G. Gato doméstico: futuro desafio para controle da raiva em áreas urbanas? Pesquisa Veterinária Brasileira, v.30, n.2, p.186-9, 2010. https://doi.org/10.1590/S0100-736X2010000200015

GÓMEZ, L.F.; ATEHORTUA, C.G.; PADILLA, S.C.O. La influencia de las mascotas en la vida humana. Revista Colombiana de Ciencias Pecuarias, v.20, n.3, p.377-86, 2007.

HARALAMBIDOU, S.; VLACHAKI, E.; IOANNIDOU, E.; MILIONI, V.; HARALAMBIDIS, S.; KLONIZAKIS, I. Pulmonary and myocardial manifestations due to Toxocara canis infection. European Journal of Internal Medicine, v.16, n.8, p.601-602, 2005. https://doi.org/10.1016/j.ejim.2005.04.008

HOFFMAN, W.A.; PONS, J.A.; JANER, J.L. Sedimentation concentration method in Schistosomiasis mansoni. The Puerto Rico Journal of Public Health and Tropical Medicine, v.9, p.283-298, 1934.

HUBER, F.; BOMFIM, T.C.B.; GOMES, R.S. Comparação entre infecção por Cryptosporidium sp. e por Giardia sp. em gatos sob dois sistemas de criação. Revista Brasileira de Parasitologia Veterinária, v.11, n.1, p.7-12, 2002. 
IBGE-Instituto Brasileiro de Geografia e Estatística. Pesquisa nacional de saúde: 2013: acesso e utilização dos serviços de saúde, acidentes e violências: Brasil, grandes regiões e unidades da federação / IBGE, Coordenação de Trabalho e Rendimento. - Rio de Janeiro: IBGE, 2015.

ISHIZAKI, M.N.; NASCIMENTO, A.A.; KANETO, C.N.; MONTANO, T.R.P.; PERRI, S.H.V.; VASCONCELOS, R.O. Frequência e intensidade parasitária de helmintos gastrintestinais de felinos da zona urbana do município de Araçatuba, SP. ARS Veterinária - Jaboticabal, v.22, n.3, p.212-216, 2006.

JAFFRY, K.T.; ALI, S.; RASOOL, A.; RAZA, A.; GILL, Z.J. Zoonoses. International Journal of Agriculture \& Biology, v.11, n.2, p.217-220, 2009.

LEE, A.C.Y.; SCHANTZ, P.M.; KAZACOS, K.R.; MONTGOMERY, S.P.; BOWMAN, D.D. Epidemiologic and zoonotic aspects of ascarid infections in dogs and cats. Trends in Parasitology, v.26, n.4, p.155-61, 2010. https://doi.org/10.1016/j.pt.2010.01.002

LEITE, L.C. Ocorrência de endoparasitas com potencial zoonótico de transmissão em fezes de gatos (Felis catus domesticus Linnaeus, 1758) domiciliados na área urbana e região metropolitana de Castro-Paraná-Brasil. Ambiência, v.8, n.3, p.923930, 2012. https://doi.org/10.5777/ambiencia.2012.05.01nt

LIMA, A.F.M.; LUNA, S.P.L. Algumas causas e consequências da superlotação canina e felina: acaso ou descaso? Revista de Educação Continuada em Medicina Veterinária e Zootecnia do CRMV-SP, v.10, n.1, p.32-38, 2012. https://doi.org/10.36440/recmvz.v10i1.258

LIMA, J.A.S.; REZENDE, H.H.A.; ROCHA, T.M.D.D.; CASTRO, A.M. Analysis of the accuracy of different laboratory methods for the diagnosis of intestinal parasites from stray and domiciled cats (Felis catus domesticus) in Goiânia, Goiás, Brazil. Brazilian Journal of Veterinary Parasitology, v.27, n.1, p.94-97, 2018. https://doi.org/10.1590/s1984-29612018004 
LIMA, F.G.; AMARAL, A.V.C.; OLIVEIRA, R.; ALVES, E.; SILVA, E.B.; TASSARA, N.; FREITAS, P.H.O.; BARBOSA, V.T. Frequência de enteroparasitas em gatos no município de Goiânia-Goiás, no ano de 2004. Enciclopédia Biosfera, v.2, n4, 4p., 2006.

MARQUES, S.M.T.; OLIVEIRA, M.R.F.; GOMES, M.J.T.M. Parasitos gastrintestinais em gatos da cidade de Porto Alegre, Rio Grande do Sul. PUBVET, v.11, n.11, p.1132-1137, 2017. https://doi.org/10.22256/pubvet.v11n11.1132-1137

MCCARTHY, J.; MOORE, T.A. Emerging helminthes zoonoses. International Journal of Parasitology, v.30, n12-13, p.1351-1360, 2000. https://doi.org/10.1016/S0020-7519(00)00122-3

MCPHERSON, C.N.L. Human behavior and the epidemiology of parasitic zoonoses. International Journal for Parasitology, v.35, n.11-12, p.1319-1331, 2005. https://doi.org/10.1016/j.ijpara.2005.06.004

MIRCEAN, V.; TITILINCU, A.; VASILE C. Prevalence of endoparasites in household cat (Felis catus) populations from Transylvania (Romania) and association with risk factors. Veterinary Parasitology, v.171, p.163-166, 2010. https://doi.org/10.1016/j.vetpar.2010.03.005

MONTEIRO, M.F.M.; RAMOS, R.A.N.; CALADO, A.M.C.; LIMA, V.F.S.; RAMOS, I.C.N.; TENÓRIO, R.F.L.; FAUSTINO, M.A.G.; ALVES, L.C. Gastrointestinal parasites of cats in Brazil: frequency and zoonotic risk. Revista Brasileira de Parasitologia Veterinária, v.25, n.2, p.254-257, 2016. https://doi.org/10.1590/S1984$\underline{29612016019}$

MORO, B.C.; PRADEBON, J.B.; SANTOS, H.T.; QUEROL, E. Ocorrência de Ancylostoma spp. e Toxocara spp. em praças e parques públicos dos municípios de Itaqui e Uruguaiana, fronteira oeste do Rio Grande do Sul. Biodiversidade Pampeana, v.6, n.1, p.25-29, 2008. 
MUNDIM, M.J.S.; SOUZA, S.Z.; HORTÊNCIO, S.M.; CURY, M.C. Frequência de Giardia sp. por duas técnicas de diagnostico em fezes de cães. Arquivo Brasileiro de Medicina Veterinária e Zootecnia, v.55, n.6, p.770-773, 2003. https://doi.org/10.1590/S0102-09352003000600016

MUNDIM, T.C.D.; OLIVEIRA JÚNIOR, S.D.; RODRIGUES, D.C.; CURY, M.C. Frequência de helmintos em gatos de Uberlândia, Minas Gerais. Arquivo Brasileiro de Medicina Veterinária e Zootecnia, v.56, n.4, p.562-563, 2004. https://doi.org/10.1590/S0102-09352004000400022

NEVES, D. P. Parasitologia Humana. São Paulo: Editora Atheneu, 2005.

PALMER, C.S.; THOMPSON, R.C.A.; TRAUB, R.J.; REES, R.; ROBERTSON, I.D. National study of the gastrointestinal parasites of dogs and cats in Australia. Veterinary Parasitology, $\quad$ v.151, n.181-190, 2008. https://doi.org/10.1016/j.vetpar.2007.10.015

PALMER, C.S.; TRAUB, R.B.; ROBERTSON, I.D.; DEVLIN, G.; REES, R.; THOMPSON, R.C. Determining the zoonotic significance of Giardia and Cryptospordium in Australian dogs and cats. Veterinary Parasitology, v.154, n.1-2, p.142-147, 2008. https://doi.org/10.1016/j.vetpar.2008.02.031

PALUDO, M.L.; FALAVIGNA, D.L.M.; GOMES, M.L. Frequência de infecção por Toxocara em crianças atendidas em serviço público de Maringá, Sul do Brasil. Revista do Instituto de Medicina Tropical de São Paulo, v.49, n.6, p.343-348, 2007. https://doi.org/10.1590/S0036-46652007000600002

PAWLOWSKI, Z. Toxocariasis in humans: clinical expression and treatment dilemma. Journal of helminthology, v.75, n.4, p.299-305, 2001. https://doi.org/10.1017/S0022149X01000464 
PIVOTO, F.L.; LOPES, L.F.D.; VOGEL, F.S.F.; BOTTON, A.S.; SANGIONI, L.A. Ocorrência de parasitos gastrointestinais e fatores de risco de parasitismo em gatos domésticos urbanos de Santa Maria, RS, Brasil. Ciência Rural, v.43, n.8, p.14531458, 2013. https://doi.org/10.1590/S0103-84782013000800018

RAGOZO, A.M.A.; SILVA, J.C.R.; CARAVIERI, R.; AMAJONER, V.R.; MAGNABOSCO, C.; GENNARI, S.M. Ocorrência de parasitos gastrintestinais em fezes de gatos das cidades de São Paulo e Guarulhos. Brazilian Journal of Veterinary Research and Animal Science, v.39, n.5, p.244-246, 2002. https://doi.org/ $\underline{10.1590 / \mathrm{S} 1413-95962002000500005}$

REY, L. Parasitologia: parasitos e doenças parasitárias do homem nos trópicos ocidentais. Rio de Janeiro: Editora Guanabara Koogan, 2008.

SANTARÉM, V.A.; GIUFFRIDA, R.; ZANIN, G.A. Larva migrans cutânea: ocorrência de casos humanos e identificação de larvas de Ancylostoma spp em parque público do município de Taciba, São Paulo. Revista Brasileira de Medicina Tropical, v.37, n2, p.179-181, 2004. https://doi.org/10.1590/S0037$\underline{86822004000200014}$

SAPORITO, L.; SCARLATA, F.; COLOMBA, C.; INFURNARI, L.; GIORDANO, S.; TITONE, L. Human toxocariasis: a report of nine cases. Acta Paediatrica, v.97, n.9, p.1301-1302, 2008. https://doi.org/10.1111/j.1651-2227.2008.00902.x

SCHANTZ, P.M. Parasitic zoonoses in perspective. International Journal for Parasitology, v.21, n.2, p.161-170, 1991. https://doi.org/10.1016/00207519(91)90006-S

SILVA, J.C.S.; COSTA, A.P.; PRASERES, D.C.; TORRES, M.A.O.; OLIVEIRANETA, M.D.; TEÓFILO, T.S. Endoparasitas em cães e gatos diagnosticados em São Luís - Maranhão. PUBVET, v.11, n.6, p.587-595, 2017. https://doi.org/10.22256/pubvet.v11n6.587-595 
SOUSA, V.R.; ALMEIDA, A.F.; CÂNDIDO, A.C.; BARROS, L.A. Ovos e larvas de helmintos em caixas de areia de creches, escolas municipais e praças públicas de Cuiabá, MT. Ciência Animal Brasileira, v.11, n.2, p.390-395, 2010. https://doi.org/10.5216/cab.v11i2.3150

THOMPSON, R.C.A.; PALMER, C.S.; HANDLEY, R.O. The public health and significance of Giardia and Cryptosporidium in domestic animals. The Veterinary Journal, v.177, n.1, p.18-25, 2007. https://doi.org/10.1016/j.tvjl.2007.09.022

TORRICO, K.J.; SANTOS, K.R.; MARTINS, T.; PAZ E SILVA, F.M.; TAKAHIRA, R.; LOPES, R.S. Ocorrência de parasitas gastrintestinais em cães e gatos na rotina do laboratório de enfermidades parasitárias da FMVZ/UNESP-BOTUCATU, SP. Revista Brasileira de Parasitologia Veterinária, v.17, Supl.1, p.182-183, 2008.

VITAL, T.E.; BARBOSA, M.R.A.; ALVES, D.S.M.M. Ocorrência de parasitos com potencial zoonótico em fezes de cães e gatos do Distrito Federal. Ensaios e Ciência: Ciências Biológicas, Agrárias e da Saúde, v.16, n.1, p.9-23, 2012.

WEESE, J.S.; PEREGRINE, A.S.; ARMSTRONG, J. Occupational health and safety in small animal veterinary practice: Part II-Parasitic zoonotic diseases. Canadian Veterinary Journal, v.43, n.10, p.799-02, 2002.

WILLIS, I.I. A simple levitation method for the detection of hookworm ova. Medical Journal of Australia, v.2, n18, p.375-376, 1921. https://doi.org/10.5694/j.1326-5377.1921.tb60654.x 